\title{
Open Science and Future Research Services for Air Transport
}

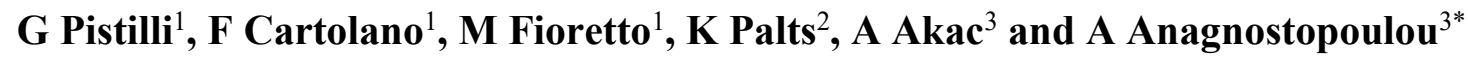 \\ ${ }^{1}$ FIT Consulting srl, Via Acciaresi Primo 8, Roma 00157, Italy \\ ${ }^{2}$ Deutsches Zentrum für Luft- und Raumfahrt e.V. (DLR), Linder Höhe, Köln 51147, \\ Germany \\ ${ }^{3}$ Centre for Research and Technology Hellas, Hellenic Institute of Transport, G.Kasimati 1 \\ str., Piraeus 18531, Greece \\ Email: a.anagnostopoulou@certh.gr(Afroditi Anagnostopoulou)
}

\begin{abstract}
.
This study aims to identify pointers about future research services, applications, regulations and policies utilizing Open Science in air transport by collecting meaningful insights from the current scientific production activities of relevant European Technology Platforms and main influential organizations of key actors in this sector. The key actors are classified into three categories: (i) industry, (ii) research community and (iii) public authorities, and their different positioning is considered based on their attributes and roles. In particular, Technology Platforms stand for industry by involving representatives of DLR and HUMANIST and the main influential organizations stand for research community and public authorities by involving representatives of EATEO and UITP, respectively. The methodology used in a bottom-up mode, moving from scenes (specific situations or circumstances involving one or more actors) to scenarios (group of more scenes) and then to use cases (more scenarios applicable to different actors) that determine needs and objectives that Open Science could
\end{abstract}


support in air transport sector. The different planning levels (strategic, tactical and operational) are considered and six competence areas (i.e., business modelling, environmental, legal/regulatory, socio-economic, technology and transport planning) set the basis of the systematic identification and analysis of use cases. Therefore, the main research trends are defined, research topics and the corresponding needs are provided as well as the proper recommendations are presented in an attempt to support future scientific work in the context of Open Science.

Keywords: scenarios, use cases, needs, recommendations.

\section{Introduction}

In recent years, European Commission has supported actions to setting up a community of transport research organizations willing to work on the basis of a commonly agreed Open Science Code of Conduct [1] with the aim to creating a solid knowledge based on the implementation of Open Science approaches in transport research. Towards this direction, the European Open Science Cloud (EOSC) [2] has been developed to enable sharing and re-use of research data across disciplines and borders, taking into account relevant legal, security and privacy issues. A number of initiatives of Open Science and FAIR (Findable, Accessible, Interoperable, and Reusable) principles have been promoted by the Horizon 2020 Work Programme 2018-2020 for the provision of a sustainable future economic growth plan [3] and nearly $10 \%$ of the budget channeled to their direct or indirect support [4].

In the context of air transport research, Open Science and FAIR principles are still at an early stage and there is lack of dissemination and exploitation achievements for the EU projects funded either by FP7 or H2020 Programmes. Therefore, the current perception of the AAT (Aeronautics \& Air Transport) community is that a balanced and sustainable concept should be adopted to allow easy and efficient access to both, breadth and depth of knowledge enhancing the role of Open Science in future European research of air transport sector. In addition, AAT understands that a broad and consistent approach and a stable access to Open Science are necessary to deliver much more stable and reliable results [5].

The European Union's INEA (Innovation and Networks Executive Agency) focused on achieving these challenges and several research projects have undertaken actions in the wider 
landscape of Open Science in aeronautics and air transport [6]. In particular, the methodology of AGILE project [7] has been implemented in AGILE's Open Access framework which collects the aircraft multidisciplinary design technologies and could be used by a broad spectrum of organizations. The AW-DRONES project [8] will develop an open repository containing structured information about technical rules, procedures and standards for drones worldwide. The Future Sky Safety project will also [9] contribute to Open Science policy through the realization of more than 30 Open Access publications, which will be made available through OpenAIRE. In addition, the OSCAR project aims to pave the way towards Open Science in European aviation research by developing, validating, and promoting a suitable Open Science concept [10].

In an attempt to exploit the new opportunities that Open Science brings in aeronautics and air transport research, this study aims to identify recommendations about future research services, applications, regulations and policies utilizing Open Science by collecting meaningful insights from the current scientific production activities of relevant European Technology Platforms and main influential organizations of key actors in this sector. The key actors could be classified into three categories: (i) industry, (ii) research community and (iii) public authorities, and their different positioning is considered based on their attributes and roles. In particular, Technology Platforms stand for industry by involving representatives of DLR and HUMANIST and the main influential organizations stand for research community and public authorities by involving representatives of EATEO and UITP, respectively.

The remainder of the paper is organized as follows: Section 2 describes the methodology followed and analyzes some indicative use cases for each of the six competence areas (i.e., business modelling, environmental, legal/regulatory, socio-economic, technology and transport planning) that cover the various aspects in air transport sector. Then, a systematic assessment is presented in Section 3 describing the main research trends, research topics and needs of key actors following a descriptive and structured mode. It is an attempt to define and classify research trends and topics as well as to identify the needs and provide the corresponding recommendations. Finally, in Section 4 conclusions are drawn and pointers for future research provided.

\section{Methodology}


The applied methodology for the overall assessment of scientific production against innovation uptakes involves a systematic review of current scientific production for air transport mode by assessing activity of relevant Technology Platforms (i.e., DLR and HUMANIST) and the main influential organizations (i.e., EATEO and UITP). It also considers positioning of different key actors based on the attributes and roles of the key actors (i.e., industry, research community, and public authorities). The used modelling approach follows a bottom-up process utilizing scenes, scenarios and use cases in air transport mode as well as interrelates the derived use cases with current research trends.

At a lowest level, a scene is defined as a situation that occurred in a particular context. Scenes show a snapshot in the considered environment and consider the relationship of all involved entities. Then, scenarios are developed. Each scenario is a chronological sequence of scenes describing a temporal development based on events and actions. Within each scenario, goals and intentions of actors get explicit. At a highest level, a use case is used to describe the purpose for which actors interact with a system to achieve a defined goal and includes several scenarios consisted of many scenes as depicted in Figure 1.

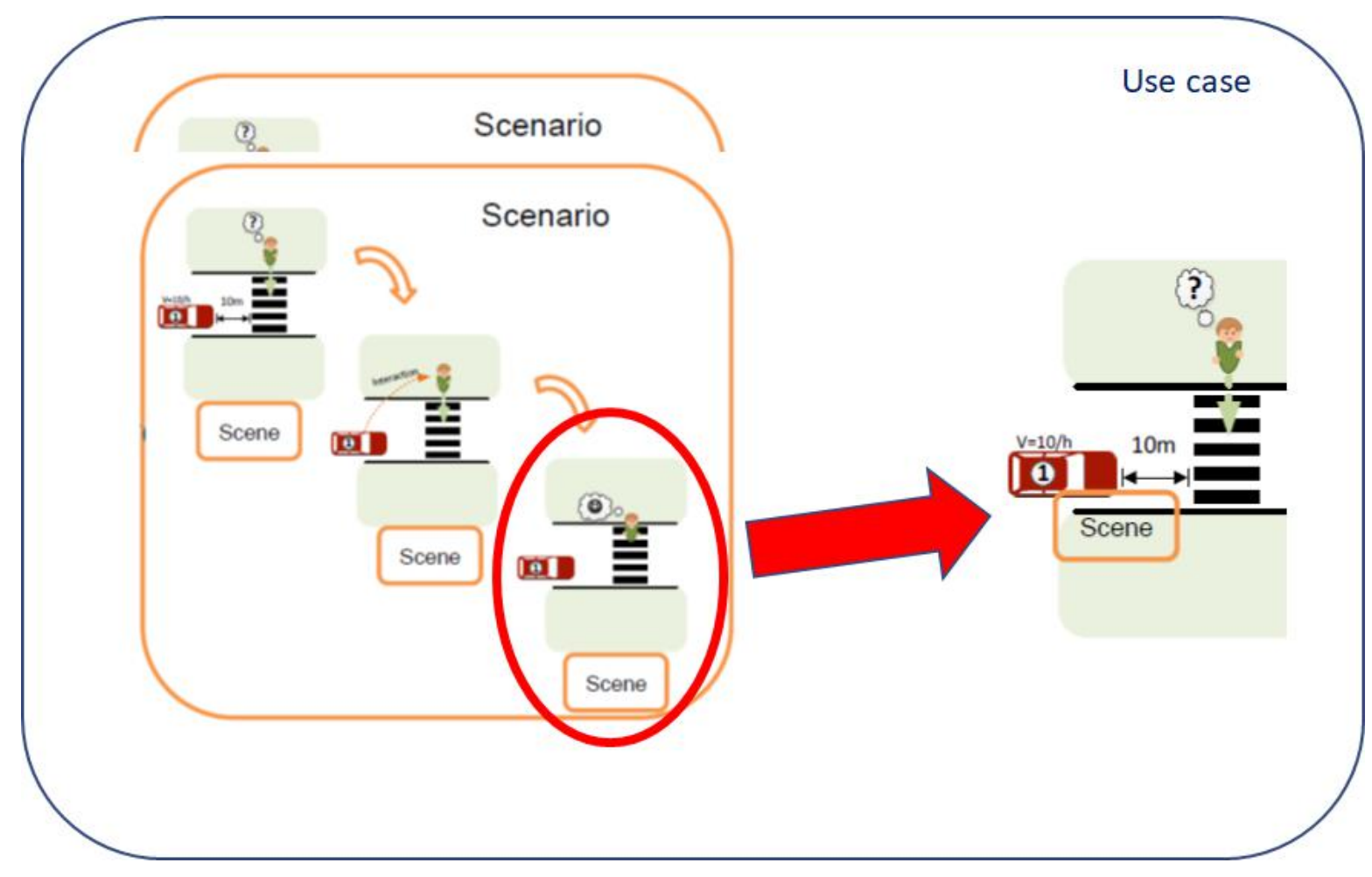

Figure 1. Use case structure 
A group of experts was engaged to provide input about the identification of research trends, research topics and needs. Their significant experience and expertise ensured the high quality of their input about current scientific production. In more detail:

- DLR research center represents more than 800 companies, from start-ups to multinationals, connecting over 50 industry sectors through a membership program designed to engage every level and discipline.

- $\quad$ HUMANIST is a French association founded from research centers, universities and SMEs active in Human Machine Interface across transport modes. In total, HUMANIST consists of 22 members from 15 countries.

- $\quad$ EATEO is a non- governmental, non- Profit Organization that provides a common forum for European aviation training and aviation education. It consists of 25 members from EU and US countries.

- UITP is an association that represents the urban, suburban and regional passenger public transport operators and organizing authorities. In total UITP consists of 1400 members from 96 countries.

The group of experts identified an indicative use case as presented in table 1 for each competence area (i.e. business modelling, environmental, legal/regulatory, socio-economic, technology and transport planning). The business modelling competence area gives a description of means and methods that a company applies to earn revenues projected in its plans. The technological area covers new technologies and innovation applied to transport for technological improvements and the environmental area delivers substantial socioeconomic benefits and at the same time lowering impacts on the environmental system. The legal/regulatory competence area covers regulations, action plans, policy instruments and incentives, and the socio-economic refers to accessibility of transport and basic services. Last but not least, the transport planning competence area applies to sustainable transport vision for reducing impact to the environment, economy and society.

Table 1. Use cases.

Competence areas

Use case

Business Modelling area Engine aircraft manufactures decide to produce electric engines for the aircrafts

Environmental area A major airport hub wants to update the pricing scheme for the use 
of its infrastructure

Legal/ Regulatory area

Transport planning area

Technological area

Socio-economic area
Air Taxi between country A and B

Aviation company wants to offer a new passenger service experience

Reduction of noise and emissions produced by aircrafts

Public authorities want to improve accessibility of population with fear of flight

To this end, a systematic assessment conducted in order to present the main research trends as identified by the group of experts, to detect corresponding research topics based on previous research programmes, and to provide needs and recommendations for future scientific work (Figure 3). The analysis is applied at different planning levels (strategic, tactical and operational) and the results are presented in Section 3.

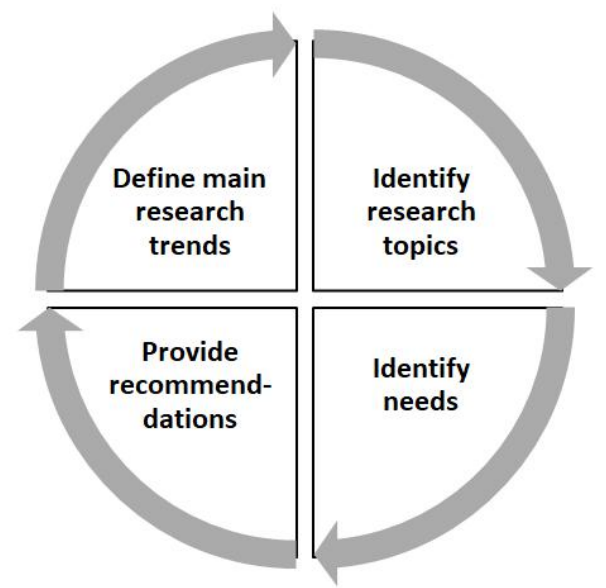

Figure 3. Steps of assessment.

\section{Results}

The main research trends per competence area are presented in table 2 and the relevant research topics presented in table 3. Research topics were defined according to previous research programmes and classified on the basis of the type of the resource used such as original research data, operational data and data from public research.

Table 2. Research trends. 


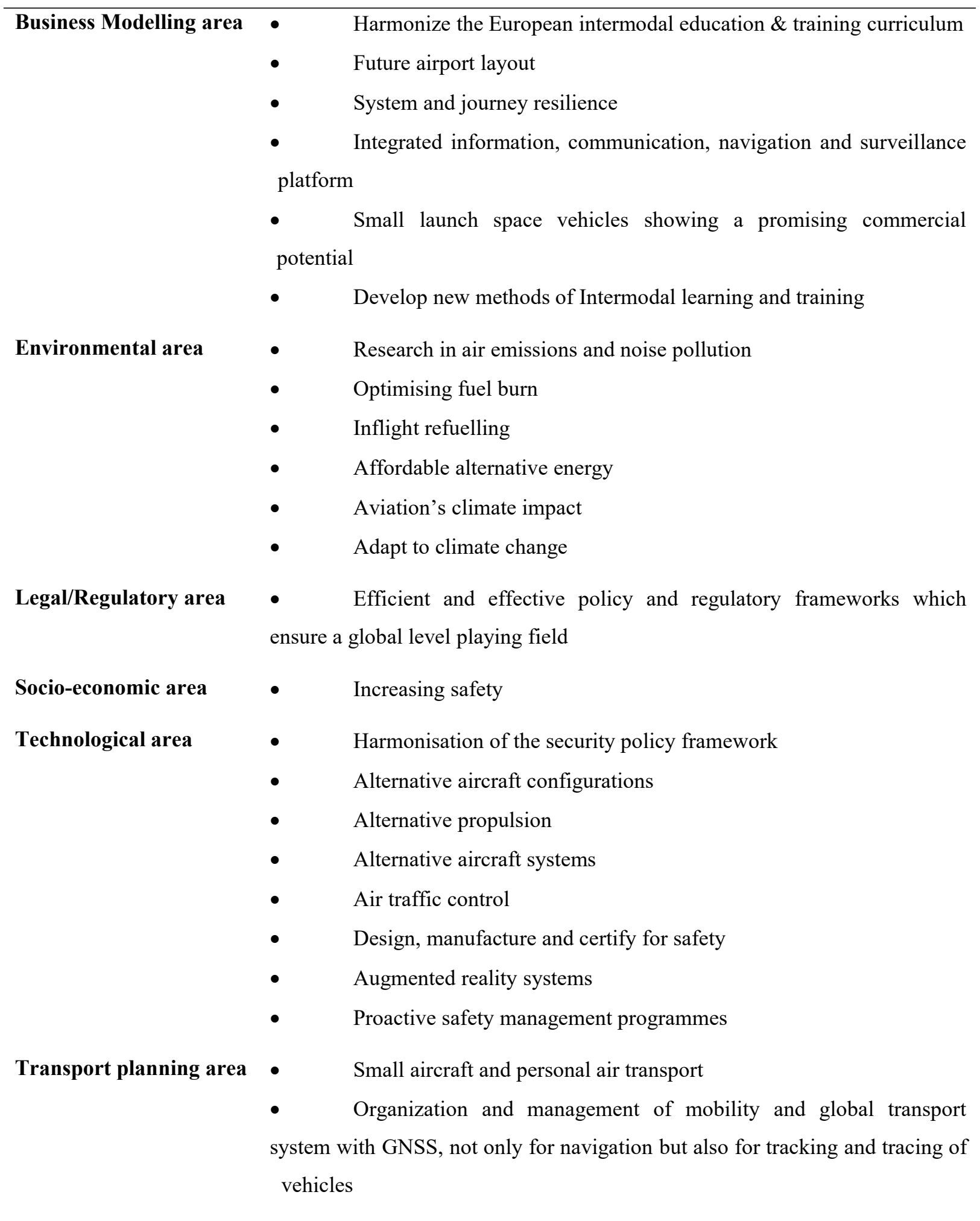

Table 3. Research topics.

Resource Research topics

\begin{tabular}{lll}
\hline Original research data & $\bullet$ & Transport regulation \\
& $\bullet$ & Integration with other transport mode
\end{tabular}




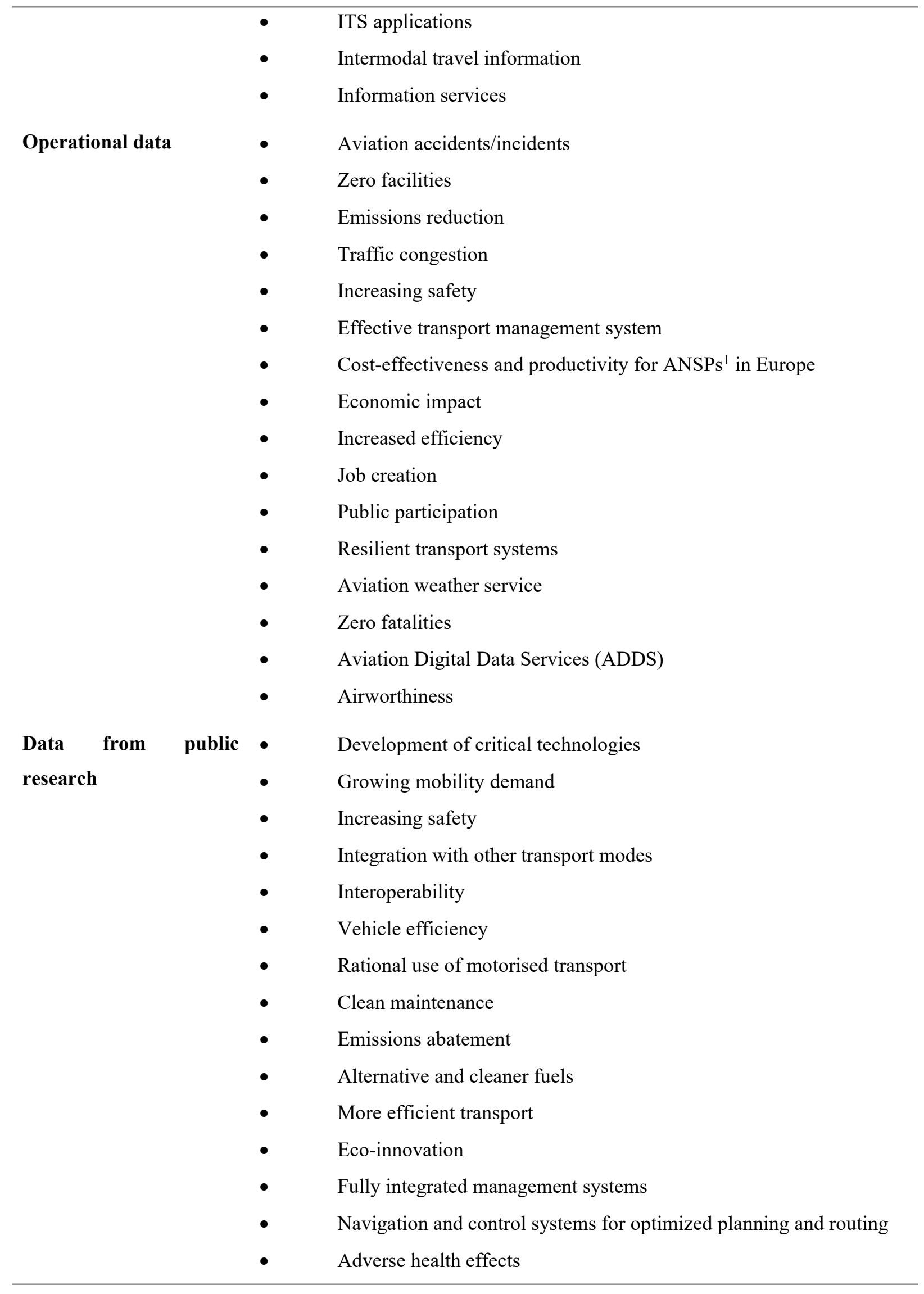

\footnotetext{
${ }^{1}$ Air Navigation Service Providers
} 


\begin{tabular}{ll}
\hline$\bullet$ & Dependence on fossil fuels \\
$\bullet$ & Competitiveness \\
$\bullet$ & Accessibility \\
- & Forward-looking activities \\
- & Air quality \\
- & Multi-disciplinary collaborations \\
$\bullet$ & New materials and processes \\
\hline
\end{tabular}

Studying the use case about business modelling area "Engine aircraft manufactures decide to produce electric engines for the aircrafts", the needs that should be addressed are (i) reorganization of the production process (e.g., time required for finishing the element, resources required, work flow), (ii) training of employees within the work time, (iii) an emergency plan with the minor losses for the company, and (iv) reconsideration of the initial decision should be considered by the company. Correspondingly, the use case of the environmental competence area is that "a major airport hub wants to update the pricing scheme for the use of its infrastructure" and the needs that should be addressed include a sustainable business plan in order to figure out how much fees should decrease for electric aircraft and how much they should be increased for conventional aircrafts. In addition, the increase margin should be decided based on the policy of the airport shareholders for covering this specific use case.

The use case of an "air taxi between country A and B" is defined in the aspect of legal/regulatory area and the derived needs include processes for informing the air traffic control when changing aerospace, tuning radio frequencies to the air traffic control of each country and finding a station to land and then to continue using a different transport mode if it is needed. In addition, the use case about transport planning area, which is "aviation company wants to offer a new passenger service experience", needs to ensure the required aircraft characteristics (e.g. number of seats, energy consumption, approximate price) and the number of required aircrafts, to examine and decide financial resources to be compromised and define contract conditions (with maintenance included in the contract or not) for the tender/leasing contract as well as to agree with the supplier the delivery conditions (e.g. place, time).

For the technological area, the use case presents the "reduction of noise and emissions produced by aircrafts" and the main need is to develop innovative aircrafts with environmentally friendly engines and low noise and emissions. Finally, the use case of 
"public authorities that want to improve accessibility of population with fear of flight" is about socio-economic competence area and several needs should be addressed. The status of airports should be assessed in terms of accessibility as well as the regulations linked to the accessibility at international, national and regional level should be checked. A proper target should be defined, a plan of measurements (tender, time frame, etc.) is required and corresponding budget is needed for executing the approved plan. Aviation companies should also be involved to buy proper aircrafts with disability seats options.

\section{Recommendations}

Studying the current research trends for air transport sector with the defined use cases, the gained results reveal that some issues have not been addressed yet and the following recommendations are proposed:

- The technological innovation should be enhanced in designing aircrafts in order to reduce the environmental impact and invest into sustainable environment.

- Cooperation between scientists and regulatory units should be fostered in order to regulate and certify new technology without jeopardizing security and safety issues.

- Fragmentation of European Sky is one of the problems to cause congestion in the airspace and therefore delays in the air traffic. Further development of Single European Sky needs to be fostered, to harmonize the procedures and therefore reduce the delays in the air traffic management.

- "Multi-modal connected" Europe needs to be created in order to fulfil passengers' needs. A build-up of passenger centric transport network, instead of transport mode centric network, could be considered.

- $\quad$ Enhancement of cyber-security and efficient data exchanges to create a more resilient and faster aviation environment.

- Foster cooperation at national and international level for the aviation sector should be achieved.

- Innovation and research in aviation sector should be supported to remain it on the competitive level.

Open Science could support the aforementioned recommendations as it aims to provide a new era in air transport research by making it more efficient and reliable. It utilizes the 
globalization and ICT developments exploiting the available open data in an attempt to achieve open scholarship or, in other words, knowledge sharing, active research outputs, and open collaboration of researchers, industry players and public authorities. Initiating the transition in Open Science, a transport research cloud is imperative in order to promote territorial and cross-border cooperation for an integrated and sustainable air transport research observatory. In this context, the TOPOS “Transport fOrum / Observatory for Promoting Open Science" has been recently developed in collaboration with OpenAIRE within the development of the BE OPEN project [11] funded from the European Union's H2020 research Programme and aims to be a portal area to gather all research results and the derived recommendations could be used for topics in the corresponding forum, in which an evidencebased discussion and cross-fertilization of ideas among researchers will be fostered.

\section{Conclusions}

The proposed recommendations, derived by the systematic assessment of use cases, provide a grid of pointers for future research services in the area of aeronautics and air transport. The main goal is to present critical issues and needs that should be considered in order to be more efficient and proactive by utilizing Open Science in air transport research. The main contribution of this study is that deals with the main research trends and the research topics of air transport and examines how the corresponding needs could be supported by Open Science exploiting previous initiatives of EU funded research projects. Furthermore, a systematic review of current scientific production of air transport has been achieved and an overall assessment against innovation uptakes are provided. Besides the aforementioned observations, consideration is also given on future research activities and directions are drawn in order to present evolutionary services based on Open Science.

\section{Acknowledgments}

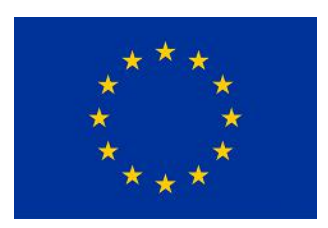

This project has received funding from the European Union's Horizon 2020 research and innovation programme under grant agreement No 824323. The results in this paper reflect only the authors' view. Neither the Innovation and Network Executive Agency nor the European Commission is responsible for any use that may be made of the information contained therein. 


\section{References}

[1] Horizon 2020 Framework Programme, TOPIC ID: MG-4-2-2018. Available at: https://ec.europa.eu/info/funding-tenders/opportunities/portal/screen/opportunities/topicdetails/mg-4-2-2018

[2] European Open Science Cloud, 2018. Available at: https://ec.europa.eu/research/openscience/pdf/eosc_strategic_implementation_roadmap_1 arge.pdf\#view $=$ fit\&pagemode $=$ none

[3] Burgelman J C, Pascu C, Szkuta K, Von Schomberg R, Karalopoulos A, Repanas K, Schouppe M 2019. Open science, open data and open scholarship: European policies to make science fit for the 21st century. Frontiers in Big Data. 243.

[4] Horizon 2020 Work Programme 2018-2020, 2018. Avaialable at: https:/ec.europa.eu/research/participants/data/ref/h2020/wp/2018-2020/main/h2020wp1820-intro_en.pdf

[5] Spieck M, Spieck S, Penzien D, Pantelakis S, Polydoropoulou P 2020. OSCAR Project "D2.2 - Open Science in aeronautics: Survey on the existing practices and expectations". Avaialable at:https://oscar-h2020.eu/sites/default/files/202101/D2.2_Open_Science_in_aeronautics_Survey_on_the existing_practices_and expectat ions.pdf

[6] Innovation and Networks Executive Agency 2021. Horizon 2020 Collaborative Aviation Research. Available at: https://ec.europa.eu/inea/sites/inea/files/aviation_brochure_2019-web.pdf

[7] Aircraft 3rd Generation Multidisciplinary Design Optimisation for Innovative Collaboration of Heterogeneous Team of Experts - AGILE Project. Available at: https://www.agile-project.eu/

[8] Contributing to Airworthiness Standards for Mass-Market Drones Project. Available at: https://www.aw-drones.eu/

[9] Future Sky Safety Project. Available at: https://www.futuresky-safety.eu/

[10] Open ScienCe Aeronautic \& Air Transport Research Project. Available at: https://oscar$\underline{\mathrm{h} 2020 . \mathrm{eu} /}$

[11] European forum and oBsErvatory for OPEN science in transport. Available at: https://beopen-project.eu/ 